\title{
Do Combined Pharmacist and Prescriber Efforts on Medication Reconciliation Reduce Postdischarge Patient Emergency Department Visits and Hospital Readmissions?
}

\author{
Michelle Baker, BScPhm1 ${ }^{1}$, Chaim M. Bell, MD, PhD2,3, Wei Xiong, MSc', Edward Etchells, MD, MSc ${ }^{3,4}$ \\ Peter G. Rossos, MD, MBA 1,3, Kaveh G. Shojania, MD ${ }^{3,4}$, Kelly Lane, BSc'1, Tim Tripp, BSc, MLIS1, Mary Lam, BSc'1, \\ Kimindra Tiwana, BScPhm¹, Derek Leong, BScPhm1', Gary Wong, BScPhm 1,5, Jin-Hyeun Huh, BScPhm, \\ Emily Musing, MHSc ${ }^{1,5}$, Olavo Fernandes, PharmD ${ }^{1,5 *}$
}

\begin{abstract}
${ }^{1}$ University Health Network, Toronto, Ontario, Canada; ${ }^{2}$ Sinai Health System, Toronto, Ontario, Canada; ${ }^{3}$ Department of Medicine-University of Toronto, Toronto Ontario, Canada; ${ }^{4}$ Sunnybrook Health Sciences Centre, Toronto, Ontario, Canada; ${ }^{5}$ Leslie Dan Faculty of Pharmacy-University of Toronto, Toronto Ontario, Canada.
\end{abstract}

BACKGROUND: Although medication reconciliation (Med Rec) has demonstrated a reduction in potential adverse drug events, its effect on hospital readmissions remains inconclusive.

OBJECTIVE: To evaluate the impact of an interprofessional Med Rec bundle from admission to discharge on patient emergency department visits and hospital readmissions (hospital visits).

METHODS: The design was a retrospective, cohort study. Patients discharged from general internal medicine over a 57-month interval were identified through administrative databases. Patients who received an enhanced, Gold level, Med Rec bundle (including both admission Med Rec and interprofessional pharmacist-prescriber collaboration on discharge Med Rec) were assigned to the intervention group. Patients who received partial Med Rec services, Silver and Bronze level, comprised the control group. The primary outcome was hospital visits within 30 days of discharge.
RESULTS: Over a 57-month period, 9931 unique patient visits ( $n=8678$ patients) met the study criteria. The main analysis did not detect a difference in 30-day hospital visits between the intervention (Gold level bundle) and control (21.25\% vs $19.26 \%$; adjusted odds ratio, 1.06 ; $95 \%$ confidence interval $[\mathrm{Cl}], 0.95-1.19)$. Propensity score adjustment also did not detect an effect $(16.7 \%$ vs $18.9 \%$; relative risk of readmission, $0.88 ; 95 \% \mathrm{Cl}, 0.59-1.32$ ).

CONCLUSION: A long-term, observational evaluation of interprofessional Med Rec did not detect a difference in 30-day postdischarge patient hospital visits between patients who received enhanced versus partial Med Rec patient care bundles. In future prospective studies, researchers could focus on evaluating high-risk populations and specific elements of Med Rec services on avoidable, medication-related hospital admissions and postdischarge adverse drug events. Journal of Hospital Medicine 2018;13:152-157. Published online first October 4, 2017. () 2018 Society of Hospital Medicine

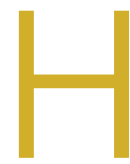

ealthcare systems are targeting effective strategies to improve patient safety and reduce hospital readmissions. Hospital readmissions can be detrimental to patients' health, a source of avoidable healthcare costs, and are frequently a reflection of the quality of patient care during transitions of care. Medication reconciliation (Med Rec) was identified as 1 of 12 interventions that may reduce 30-day readmissions; however, rigorously designed studies are scarce. ${ }^{1,2}$ Published systematic reviews and meta-analyses have

\footnotetext{
*Address for correspondence: Olavo Fernandes, PharmD, Toronto Western Hospital, University of Toronto, University Health Network, 399 Bathurst Street, Fell Pavillion, $4^{\text {th }}$ Floor, Room 200, Toronto, Ontario, M5T 2S8; Telephone: 416603-5800x 3443; Fax: 416-603-5186; E-mail: olavo.fernandes@uhn.ca

Additional Supporting Information may be found in the online version of this article
}

Received: March 8, 2017; Revised: June 22, 2017; Accepted: July 23, 2017

2018 Society of Hospital Medicine DOI 10.12788/jhm.2857 produced mixed conclusions regarding the impact of Med Rec on unplanned 30-day readmissions. ${ }^{2-4}$

In several studies, researchers have established the positive impact of Med Rec on reducing patient medication discrepancies and potential adverse drug events. ${ }^{4-8}$ Pharmacy-led Med Rec interventions have been shown to easily identify more clinically relevant and higher impact medication discrepancies when compared to usual care. ${ }^{8}$ In a systematic review, Mueller et al. ${ }^{2}$ suggest that there are several interrelated elements that determine if a Med Rec intervention will influence hospital readmissions. These elements form a multicomponent "bundle" of interventions, including a systematic medication history process, admission reconciliation, patient education on discharge, discharge reconciliation, and communication to outpatient providers. ${ }^{9}$ Several prospective randomized controlled studies have demonstrated lower readmission rates and fewer visits to the emergency department (ED) after implementing a comprehensive, interprofessional, bundled intervention (including Med Rec) from admission to discharge. ${ }^{10-13}$ A 2016 systematic 
TABLE 1. Varying Levels of Intensity (Taxonomy) of Med Rec Care Integrated With Interprofessional Medication Management

\begin{tabular}{|c|c|c|c|}
\hline Care Bundle Category & $\begin{array}{l}\text { Care Level } \\
\text { of Intensity }\end{array}$ & Key Components & Published Examples \\
\hline \multirow[t]{2}{*}{ Partial } & Bronze & $\begin{array}{l}\text { BPMH with admission reconciliation } \\
\text { Med Rec informatics platform to support the healthcare team }\end{array}$ & $\begin{array}{l}\text { Cornish et al. } 2005^{16} ; \text { Kwan et } \\
\text { al. } 2007^{17}\end{array}$ \\
\hline & Silver & $\begin{array}{l}\text { Bronze plus } \\
\text { Prescriber-only discharge Med Rec }\end{array}$ & $\begin{array}{l}\text { Wong et al. } 2008^{6} \text {; Schnipper et } \\
\text { al. } 2009^{7}\end{array}$ \\
\hline Enhanced & Gold & $\begin{array}{l}\text { Silver plus } \\
\text { Interprofessional (prescriber and pharmacist collaboration) discharge Med Rec } \\
\text { Varying degrees of medication management and pharmaceutical care }\end{array}$ & $\begin{array}{l}\text { Schnipper et al. } 2009^{7} \text {; Cesta et } \\
\text { al. } 2006^{15} \text {; Dedhia et al. } 2009^{18}\end{array}$ \\
\hline \multirow[t]{2}{*}{ Intensive } & Platinum & $\begin{array}{l}\text { Gold plus } \\
\text { Patient medication education prior to discharge (including discussion of medication changes) } \\
\text { Provision of patient-friendly reconciled medication schedules upon discharge } \\
\text { Broader attention to medication management and pharmaceutical care with pharmacist inpatient rounding }\end{array}$ & $\begin{array}{l}\text { Makowsky et al } 2009^{13} \text {; Dedhia et } \\
\text { al. } 2009^{18} \text {; Murphy et al. 2009'; } \\
\text { Nazareth et al. } 2001^{20} \text {; Al-Rashed } \\
\text { et al. } 2002^{21}\end{array}$ \\
\hline & Diamond & $\begin{array}{l}\text { Platinum plus } \\
\text { Postdischarge follow-up phone call to patient by hospital clinician (eg, nurse or pharmacist) } \\
\text { Communication of medication changes with rationale directly to community pharmacy and primary care physician }\end{array}$ & $\begin{array}{l}\text { Karapinar-Çarkit et al. 20095; } \\
\text { Jack et al 200911; Gillespie et al. } \\
2009^{12} \text {; Schnipper et al. } 2006^{22} \\
\text { Walker et al. } 2009^{23}\end{array}$ \\
\hline \multicolumn{4}{|c|}{$\begin{array}{l}\text { NOTE: Table } 1 \text { outlines a proposed continuum of degrees of Med Rec care bundles varying from Bronze to Silver, Gold, Platinum, and Diamond. The key being that more advanced levels of } \\
\text { care and higher intensities have a progression in the care elements in the multicomponent bundle: true interprofessional collaboration, active patient and family participation in all stages, and } \\
\text { the comprehensive nature of transition communication personalized for more providers. Of note, for a given ward and interprofessional team, different proportions of patients may receive levels } \\
\text { from Bronze to Platinum and the quality and accuracy of each stage may also vary. The degree on the care level intensity continuum has a meaningful differential impact on patient outcomes } \\
\text { (such as hospital readmissions) as demonstrated in published studies. Adapted with permission from Healthcare Quarterly 2012;15(Special Issue):44. }\end{array}$} \\
\hline
\end{tabular}

review and meta-analysis specifically evaluated pharmacy-led Med Rec programs (the majority of which included interventions involving multicomponent bundles) and demonstrated a significant reduction in posthospital healthcare utilization. ${ }^{14}$

Although comprehensive, interprofessional, bundled interventions have been shown to reduce readmission rates and ED visits in randomized controlled trials (RCTs), limited resources often prevent hospitals from consistently implementing all aspects of these multicomponent interventions. In practice, clinicians may provide varying components of the bundle, such as the combination of admission medication history by the pharmacist and discharge Med Rec completed by the physician alone. The unique impact of combined pharmacist and prescriber Med Rec interventions from admission to discharge on readmissions remains inconclusive. Further, it is unclear which high-risk patient groups will benefit the most from these interventions. We set out to evaluate the impact of an enhanced, interprofessional Med Rec process from admission to discharge (characterized within the context of a novel taxonomy continuum that specifies clinician involvement and intensity of services) on readmissions to hospital and ED visits within 30 days of discharge.

\section{METHODS}

We conducted a retrospective, observational, analytical cohort study using QuadraMed's Computerized Patient Record and the EMITT (Electronic Medication Information Transfer Tool) ${ }^{15}$ to collect data from 2007 to 2011.

\section{Setting}

The study was conducted at a 417-bed tertiary care teaching hospital in Toronto, Ontario, Canada.

\section{Med Rec Process and Description of Exposure (Intervention)}

The targeted clinical areas had sustained interprofessional models of patient care in place from admission to discharge. They also were actively using an in-house EMITT to facilitate the documentation and tracking of Med Rec efforts throughout patient admission, transfer, and discharge.${ }^{15}$ On admission, the pharmacist conducted a best possible medication history (BPMH). A BPMH provides the cornerstone for Med Rec. It differs from a routine medication history in that it involves (1) a systematic process for interviewing the patient (or family) and (2) a review of at least one other reliable source of information (eg, a provincial medication database, an inspection of medication vials, or contact with the community pharmacy) to obtain and verify patient medications (prescribed and nonprescribed). The pharmacist recorded the BPMH in the electronic patient record. The application supported admission and discharge Med Rec. On discharge, there were 2 options: (1) the prescriber alone would review and complete the discharge Med Rec and generate electronic prescriptions (Table 1, Silver level care) or (2) the pharmacist would collaborate with the prescriber to complete the discharge reconciliation and the prescriber would electronically generate prescriptions (Table 1, Gold level care). All clinical areas had a combined pharmacist and prescriber Med Rec model in place at admission, but the proportion of patients receiving discharge reconciliation completed by pharmacist and prescriber versus the prescriber-alone varied based on the individual clinician's practices.

\section{Patient Selection}

All consecutive hospitalized patients admitted and discharged by the general internal medicine [GIM] service from March 
2007 to December 2011 were included. The GIM service was chosen for the main analysis because they had been performing the intervention for the longest period of time and had the largest population of patients. Patients were identified via their hospital-specific medical record identification number and specific hospital-visit number. Patients were excluded if any of the following occurred: (1) the length of stay of their index admission was less than 24 hours; (2) they died during the visit; (3) they were transferred to a separate acute care inpatient facility; or (4) they left hospital against medical advice. Patient visits were excluded as index cases from the analysis if they were returning within 90 days of a previous discharge.

\section{Outcomes}

The primary study outcome was the occurrence of an inpatient readmission or ED visit within 30 days of discharge. In our secondary analyses, we examined the impact of the intervention on high-risk patient populations, such as those $\geq 65$ years of age, with a length of stay, acuity of admission, Charlson comorbidity index, and emergency department visits in past 6 months (LACE) index score $\geq 10$ (see supplementary Appendix 1 for LACE score description), on high-alert medications (1 or more of warfarin, insulin, digoxin, and opioids), and on $\geq 10$ medications.

\section{Data Collection}

\section{Identification of Exposure of Interest}

We used the electronic database to capture all patients who received pharmacist and prescriber supported admission-to-discharge reconciliation. We explicitly defined increasing intensity of Med Rec care in categories of Bronze, Silver, and Gold care levels (Table 1). The exposed (intervention) group received an enhanced Med Rec bundle (patients receiving Gold level care). The control group was made of patients receiving a partial Med Rec Bundle (patients receiving Silver or Bronze level of care or below).

\section{Determination of Hospital Visits}

A search of administrative databases was used to determine if patients admitted to the targeted services had an ED visit or urgent inpatient admission to the study hospital within 30 days.

\section{Statistical Analysis}

A logistic regression for outcomes was performed. This yielded an adjusted odds ratio with a 95\% confidence interval (Cl) between the intervention and control groups. Statistical significance was determined with a 2-sided $\alpha$ level of 0.05 . In the analysis, we used Statistical Analysis Software version 9.2.

In our multivariate logistic regression model, we adjusted for confounding factors that might influence the patients' risk of readmission or the type of Med Rec they received upon discharge. By using administrative databases, patient level demographics, and the Charlson comorbidity index, the most responsible diagnosis and disease burden were collected. Medication-related factors collected included the number of medications on discharge and the presence of predefined high-alert medications. The number of medications on the medication discharge list was determined by using the electronic database. The final adjustment model included age, gender, the number of medications on discharge, and the LACE index score (supplementary Appendix 1). The LACE index score has been validated in Ontario, Canada, populations to quantify the risk of death or unplanned readmission within 30 days of discharge. ${ }^{24}$

\section{Propensity Score Adjustment}

Propensity scoring (probability of treatment assignment conditional on observed baseline characteristics) was planned a priori to account for possible factors that would impact whether a patient received the intervention or control care levels. The propensity score for receiving Med Rec was computed from a logistic model using Med Rec as the outcome. A structured iterative approach was used to refine this model to achieve covariate balance within the matched pairs. Covariate balance was measured by the standardized difference, in which an absolute standardized difference $>10 \%$ represents meaningful imbalance. ${ }^{25}$ From the original cohort, we attempted to match patients who had the intervention to patients from the control by means of a matching algorithm using the logit of the propensity score for receiving the intervention. ${ }^{26}$

\section{Subgroup Analysis}

We also examined the impact of the intervention on high-risk patient populations such as those $\geq 65$ years of age, with a LACE index score $\geq 10$, on high-alert medications, and on $\geq 10$ medications. A univariate analysis was conducted to identify patient-related risk predictors that may be independently correlated with a higher risk of hospital visits.

\section{RESULTS}

\section{Baseline Characteristics}

A total of 8678 patients representing 9931 unique visits met the inclusion criteria for analysis. There were 2541 unique visits (approximately $26 \%$ of visits) in the intervention group that received Gold level care and 7390 unique visits in the control group. The patients in the control group were largely patients who received the original standard of care at the institution, Silver level care (67\% of the control group). Patients who received Bronze level care or less comprised $33 \%$ of the control group.

Patients in the intervention group were significantly older (average of 68 years old versus 64 years old) and on more medications. They also notably had a longer duration of stay in hospital, an increased percentage of visits with a LACE index score $\geq 10$, and were more likely to be discharged home on a high-alert medication and with supports (Table 2).

\section{Main Analysis}

The main unadjusted analysis of GIM patients ( $n=9931$ visits) did not detect a difference in 30-day ED visits and readmissions between the intervention group (540 out of $2541 ; 21.2 \%$ ) and control (1423 out of 7390; $19.3 \%$; Table 3). By using a multivariate logistic regression model to account for age, sex, LACE index, 
TABLE 2. Baseline Characteristics of Visits: General Internal Medicine

\begin{tabular}{lcc} 
Baseline Demographics & Interprofessional Discharge Reconciliation & Did Not Receive Interprofessional Discharge Reconciliation \\
Total Visits $\mathbf{N}=\mathbf{9 9 3 1}$ & $\mathbf{N}=\mathbf{2 5 4 1}=\mathbf{7 3 9 0}$ & $64.66(19.36)$ \\
\hline Age in years at index visit, mean (SD) & $68(17.91)$ & $3540(48)$ \\
\hline Female, no. (\%) & $1200(47)$ & $8.70(14.87)$ \\
\hline Mean LOS [acute + awaiting long-term care], days (SD) & $11.47(27.19)$ & $0.52(1.18)$ \\
\hline Mean Charlson comorbidity score, no. (SD) & $0.57(1.16)$ & $8.06(2.47)$ \\
\hline Mean LACE index, no. (SD) & $8.59(2.45)$ & $1964(27)$ \\
\hline LACE index score $\geq 10$, no. (\%) & $852(34)$ & $8.72(5.63)$ \\
\hline Mean number of medications on discharge, no. (SD) & $10.17(5.10)$ & $3070(41)$ \\
\hline Greater than or equal to 10 discharge medications, no. (\%) & $1303(51)$ & $1647(22)$ \\
\hline Discharge code: home with supports, no. (\%) & $778(31)$ & $1317(18)$ \\
\hline Predefined high alert medication, no. (\%) & $604(24)$ &
\end{tabular}

NOTE: Abbreviations: LACE, length of stay ("L"), acuity of the admission ("A"), comorbidity of the patient (measured with the Charlson comorbidity index score) ("C"), and emergency department use of patients ("E"), LOS, length of stay; SD, standard deviation.

TABLE 3. Main Analysis of 30-Day Hospital Visits for General Internal Medicine

\begin{tabular}{|c|c|c|c|c|c|c|}
\hline \multirow[b]{2}{*}{ Type of Hospital Visit } & \multicolumn{2}{|c|}{$\begin{array}{c}\text { Control } \\
\text { Total Visits N = } 7390\end{array}$} & \multicolumn{2}{|c|}{$\begin{array}{c}\text { Intervention } \\
\text { Total Visits N = } 2541\end{array}$} & \multicolumn{2}{|c|}{ Total } \\
\hline & $\mathrm{n}$ & $\%$ & $\mathrm{n}$ & $\%$ & N & $\mathrm{AOR}^{\mathrm{a}}(95 \% \mathrm{Cl})$ \\
\hline ED Visit & 1352 & 18.29 & 523 & 20.58 & 1875 & $1.08(0.96-1.21), P=.18$ \\
\hline IP admission & 904 & 12.23 & 365 & 14.36 & 1269 & $1.20(1.06-1.37), P=.18$ \\
\hline ED or IP & 1423 & 19.26 & 540 & 21.25 & 1963 & $1.06(0.945-1.19), P=.33$ \\
\hline
\end{tabular}

${ }^{a} A O R$ is the adjusted odds ratio $P$ value determined by using a multivariate logistic regression model to account for age, sex, LACE index, and number of medications on discharge. NOTE: Abbreviations: AOR, adjusted odds ratio; Cl, confidence interval; ED, emergency department; IP, inpatient; LACE, length of stay ("L"), acuity of the admission ("A"), comorbidity of the patient (measured with the Charlson comorbidity index score) (" $\mathrm{C}$ "), and emergency department use of patients (" $E$ ")

and number of medications on discharge, the adjusted odds ratio was $1.06(95 \% \mathrm{Cl}, 0.95-1.19 ; P=.33)$. After propensity score adjustment, the relative risk of readmission was $0.88(16.7 \%$ vs $18.9 \% ; 95 \% \mathrm{Cl}, 0.59-1.32 ; P=.54)$.

\section{Secondary Analyses}

In each predefined high-risk patient subgroup (age $\geq 65$, LACE index score $\geq 10$, number of discharge medications $\geq 10$, and the presence of high-alert medications), analyses of our primary endpoint did not detect significant adjusted odds ratios (Table 4). In our univariate analysis, increasing number of medications, LACE index score, and male gender were independently correlated with a higher risk of hospital visits (supplementary Appendix 2).

\section{DISCUSSION}

Med Rec is widely recommended as a patient safety strategy to prevent clinically significant medication discrepancies at transitions in care. ${ }^{4.9}$ However, Med Rec varies widely in terms of what it entails and who delivers it, with the preponderance of evidence suggesting an impact on clinically significant medication discrepancies only when interprofessional care delivered includes a central role for pharmacists. ${ }^{27}$ Furthermore, Med Rec appears to impact short term readmissions only when embedded in a broader, multifac- eted, bundled intervention in which pharmacists or other team members educate patients about their medications and deliver postdischarge follow-up phone calls. ${ }^{10-13}$

As very few hospitals have the resources to sustainably deliver intensive care bundles that are represented in RCTs (characterized by Platinum and Diamond levels of care in Table 1), in our observational study, we sought to explore whether a resource-attainable, enhanced Med Rec care bundle (Gold level) had an impact on hospital utilization compared to partial Med Rec care bundles (Bronze and Silver levels). In our findings, we did not detect a significant difference on ED visits and readmissions within 30 days between enhanced and partial care bundles. In a secondary analysis of the influence of the intervention on prespecified high-risk patient subgroups, we also did not detect a difference.

As far as we are aware, our long-term, observational study is the largest to date to explore a real-life, enhanced Med Rec intervention and examine its impact on meaningful patient outcomes. We extrapolated that our intervention group received several critical attributes of a successful bundle as discussed by Mueller in a systematic review. ${ }^{2}$ Our intervention included the following: (1) a systematic BPMH process on admission; (2) integrated admission-to-discharge reconciliation processes; (3) discharge delineation of medication changes since admission; 
TABLE 4. ED or IP Admissions in High Risk Patient Subgroups-General Internal Medicine

ED Visits or Inpatient Admissions

\begin{tabular}{|c|c|c|c|c|c|c|}
\hline \multirow[b]{2}{*}{ High-risk Group } & \multirow[b]{2}{*}{$\begin{array}{l}\text { Total No. } \\
\text { of Visits }\end{array}$} & & \multirow[b]{2}{*}{ AOR $(95 \% \mathrm{Cl})$} \\
\hline & & No. of control visits & $\begin{array}{l}\text { ED or IP admissions } \\
\text { (\% of total control visits) }\end{array}$ & $\begin{array}{c}\text { No. of } \\
\text { intervention visits }\end{array}$ & $\begin{array}{c}\text { ED or IP admissions } \\
\text { (\% of total intervention visits) }\end{array}$ & \\
\hline Age $\geq 65$ & 5667 & 4087 & $787(19.26)$ & 1580 & 347 (21.96) & $1.41(0.99-1.32)$ \\
\hline No. of medications $\geq 10$ & 4373 & 3070 & $659(21.47)$ & 1303 & $285(21.87)$ & $1.00(0.85-1.17)$ \\
\hline On high-alert medication & 8010 & 6073 & $1166(19.20)$ & 1937 & $407(21.01)$ & $1.05(0.92-1.19)$ \\
\hline
\end{tabular}

NOTE: Abbreviations: AOR, adjusted odds ratio; $C l$, confidence interval; ED, emergency department; IP, inpatient; LACE, length of stay ("L"), acuity of the admission (" $\mathrm{A}$ "), comorbidity of the patient (measured with the Charlson comorbidity index score) ("C ${ }^{\prime \prime}$ ), and emergency department use of patients (" $E$ ")

(4) pharmacist involvement in reconciliation from admission to discharge; (5) an electronic platform; and (6) formal discharge reconciliation with interprofessional collaboration. Additional components in the bundle described by Mueller included the following: patient education at discharge, postdischarge communication with the patient, and communication with outpatient providers and medication management.

In our results, we did not find a difference in outcomes between the intervention and control groups. Therefore, it is possible that the enhanced bundle's focus on interprofessional involvement in discharge reconciliation (Gold care level) has no impact on hospital utilization compared to partial care bundles (Silver and Bronze levels). Kwan et al. ${ }^{3}$ describe similar findings in their systematic review, in which they evaluated the effects of hospital-based Med Rec on unintentional discrepancies with nontrivial risks for harm to patients on 30-day postdischarge hospital visits. Kwan et al. ${ }^{3}$ concluded that larger well-designed studies are required to further evaluate this outcome, but authors of current published studies suggest that Med Rec alone probably does not reduce postdischarge hospital utilization within 30 days. Med Rec may have a more significant impact on utilization when bundled with other interventions that improve discharge coordination. ${ }^{3}$

There may be several reasons why we were unable to detect a significant difference between the intervention and control groups. One limitation is that our nonrandomized, retrospective design may have led to unmeasured confounders that impacted allocation into the intervention group versus the control group. It was notable that patients in the intervention group had an increased age, longer duration of hospital stay, more medications, and high-alert medications on discharge compared to the control group and that may have biased our results towards the null hypothesis. Although the propensity score analysis attempted to adjust for this, it also did not detect a significant difference between groups.

In addition, the existing standard of care during the study period allowed for patients in the control group to receive varying levels of Med Rec. Ideally, we would have compared the intervention to a placebo group that did not receive any Med Rec-related care elements. However, as this was a real-life observational study, the majority of patients received some Med Rec services as a part of the standard of care. As a result, $67 \%$ of patients in the control group received Silver level Med $\mathrm{Rec}$ with a BPMH, admission reconciliation, and prescriber-on- ly discharge reconciliation. This may have made it more difficult to show an incremental benefit on readmissions between the intervention and control.

Also, our primary outcome of all-cause ED or hospital readmissions within 30 days may not have been sensitive enough to detect the effect of Med Rec interventions alone. Only a small proportion of readmissions within 30 days of discharge are preventable and many patient and community level factors responsible for readmissions cannot be controlled by the hospital's actions. ${ }^{28}$ Comprehensive pharmacy interventions have demonstrated decreased hospitalizations and emergency visits at 12 months; however, the largest impact was seen on the more specific outcome of medication-related hospitalizations (80\% reduction). ${ }^{29}$ Lastly, another limitation was that we were unable to capture hospital visits to other centres. However, in our region, almost $75 \%$ of readmissions are to the same site as the initial hospitalization. ${ }^{30}$

Overall, our findings in this study and novel characterization of Med Rec services are relevant to many hospital sites that are striving to implement integrated Med Rec with limited healthcare resources. Although interprofessional Med Rec likely reduces clinically significant medication discrepancies, enhanced interprofessional Med Rec on discharge (Gold Med Rec) alone may not be enough to impact hospital utilization compared to partial Med Rec services (Silver and Bronze Med Rec). Further research into practical, targeted Med Rec bundles on more specific outcomes (such as preventable postdischarge adverse events, "avoidable" hospital readmissions, and medication-related readmissions) may detect a significant benefit.

\section{CONCLUSION}

A long-term observational evaluation of interprofessional Med Rec did not detect a difference in 30-day postdischarge patient hospital visits between patients who received enhanced versus partial Med Rec patient care bundles. Researchers of future prospective studies could focus on evaluating high-risk populations or specific elements of Med Rec services on avoidable medication-related hospital admissions and postdischarge adverse drug events.

\section{Acknowledgments}

The authors thank Nita Dhir, MBA.

Presented as a poster and oral presentation at the 2012 American College of Clinical Pharmacy Annual Meeting, Hollywood, Florida, October 21-24, 2012, 
and as an encore poster presentation at the Canadian Society of Hospital Pharmacists Professional Practice Conference, Toronto, Canada, Feb 3, 2013.

Disclosure: The authors declare no conflicts of interest related to the manuscript submitted.

All monies used for the research came from the University Health Network Department of Pharmacy Budget, including the pharmacy residency program.

\section{References}

1. Hansen LO, Young RS, Hinami K, Leung A, Williams MV. Interventions to reduce 30-day rehospitalization: a systematic review. Ann Intern Med. 2011; 155:520-528.

2. Mueller SK, Sponsler KC, KripalaniS, Schnipper JL. Hospital-based medication reconciliation practices: a systematic review. Arch Intern Med. 2012;172(14): 1057-1069.

3. Kwan JL, Lo L, Sampson M, Shojania KG. Medication reconciliation during transitions of care as a patient safety strategy: a systematic review. Ann Intern Med. 2013;158:397-403.

4. Safer Health Care Now. Medication Reconciliation in Home Care Getting Started Kit. March 2015. www.ismp-canada.org/download/MedRec/Medrec_HC_English_GSK_v2.pdf. Accessed August 22, 2017.

5. Karapinar-Çarkit F, Borgsteede SD, Zoer J, Smit HJ, Egberts AC, van den Bemt PM. Effect of medication reconciliation with and without patient counseling on the number of pharmaceutical interventions among patients discharged from the hospital. Ann Pharmacother. 2009;43(6):1001-1010.

6. Wong JD, Bajcar JM, Wong GG, et al. Medication reconciliation at hospita discharge: evaluating discrepancies. Ann Pharmacother. 2008;42(10):13731379

7. Schnipper JL, Hamann C, Ndumele CD, et al. Effect of an electronic medication reconciliation application and process redesign on potential adverse drug events: a cluster-randomized trial. Arch Intern Med. 2009;169(8):771780 .

8. Mekonnen AB, McLachlan AJ, Brien JA. Pharmacy-led medication reconciliation programmes at hospital transitions: a systematic review and meta-analysis. J Clin Pharm Ther. 2016;41(2):128-144.

9. Kaboli PJ, Fernandes O. Medication reconciliation: moving forward. Arch Intern Med. 2012:172(14):1069-1070.

10. Koehler BE, Richter KM, Youngblood L, et al. Reduction of 30-day postdischarge hospital readmission or emergency department (ED) visit rates in high-risk elderly medical patients through delivery of a targeted care bundle. J Hosp Med. 2009:4:211-218.

11. Jack BW, Chetty VK, Anthony D, et al. A reengineered hospital discharge program to decrease rehospitalization: a randomized trial. Ann Intern Med. 2009;150:178-187.

12. Gillespie U, Alassaad A, Henrohn D, et al. A comprehensive pharmacist intervention to reduce morbidity in patients 80 years or older. Arch Intern Med. 2009:169(9):894-900.

13. Makowsky MJ, Koshman SL, Midodzi WK, Tsuyuki RT. Capturing outcomes of clinical activities performed by a rounding pharmacist practicing in a team environment: the COLLABORATE study [NCT00351676]. Med Care. 2009;47(6):642-650.

14. Mekonnen AB, McLachlan AJ, Brien JA. Effectiveness of pharmacist-led medication reconciliation programmes on clinical outcomes at hospital transitions: a systematic review and meta-analysis. BMJ Open. 2016;6(2):e010003.

15. Cesta A, Bajcar JM, Ong SW, Fernandes OA. The EMITT study: development and evaluation of a medication information transfer tool. Ann Pharmacother. 2006:40(6):1074-1081

16. Cornish $P$, et al. Unintended medication discrepancies at the time of hospital admission. Arch Internal Medicine, 2005, Feb: 165: 424-29.

17. Kwan Y, Fernandes OA, Nagge JJ, et al. Pharmacist medication assessments in a surgical preadmission clinic. Arch Intern Med. 2007;167(10):1034-1040

18. Dedhia $P$, Kravet $S$, Bulger J, et al. A quality improvement intervention to facilitate the transition of older adults from three hospitals back to their homes. J Am Geriatr Soc. 2009;57:1540-1546.

19. Murphy EM, Oxencis CJ, Klauck JA, et al. Medication reconciliation at an academic medical center: implementation of a comprehensive program from admission to discharge. Am J Health Syst Pharm. 2009;66:2126-31

20. Nazareth I, Burton A, Shulman S, Smith P, Haines A, Timberal H. A pharmacy discharge plan for hospitalized elderly patients - a randomized controlled trial. Age and Ageing. 2001;30(1):33-40.

21. Al-Rashed SA, Wright DJ, Roebuck N, et al. The value of inpatient pharmaceutical counselling to elderly patients prior to discharge. Br J Clin Pharmacol. 2002 Dec;54(6):657-64.

22. Schnipper JL, Kirwin JL, Cotugno MC, et al. Role of pharmacist counseling in preventing adverse drug events after hospitalization. Arch Intern Med. 2006 Mar 13;166(5):565-71.

23. Walker PC, Bernstein SJ, Jones JN, et al. Impact of a pharmacist-facilitated hospital discharge program: a quasi-experimental study. Arch Intern Med. 2009 Nov 23;169(21):2003-10.

24. van Walraven C, Dhalla IA, Bell C, et al. Derivation and validation of an index to predict early death or unplanned readmission after discharge from hospital to the community. CMAJ. 2010;182(6):551-557.

25. Normand ST, Landrum MB, Guadagnoli $E$, et al. Validating recommendations for coronary angiography following an acute myocardial infarction in the elderly: a matched analysis using propensity scores. J Clin Epidemiol. 2001;54(4):387-398.

26. Rosenbaum PR., Donald BR. Constructing a control group using multivariate matched sampling methods that incorporate the propensity score. Am Stat. 1985;39(1):33-38.

27. Fernandes $O$, Shojania KG. Medication reconciliation in the hospital: what, why, where, when, who and how? Healthc Q. 2012;15(Special Issue):42-49.

28. Joynt $K E$, Jha $A K$. Thirty-day readmissions - truth and consequences. $N$ Engl J Med. 2012;366(15):1366-1369.

29. Zed PJ, Abu-Laban RB, Balen RM, et al. Incidence, severity and preventability of medication-related visits to the emergency department: a prospective study. CMAJ. 2008:178(12):1563-1569.

30. Gruneir A, Dhalla IA, van Walraven C, et al. Unplanned readmissions after hospital discharge among patients identified as being at high risk for readmission using a validated predictive algorithm. Open Med. 2011;5(2):e104-e111. 